Aus der Klinik für venerische und Hautkrankheiten der militär-medizinischen Akademie zu St. Petersburg.

(Prof. T. P. Pawlow.)

\title{
Zur Pathologie der Pityriasis rubra pilaris.
}

Von

Privatdozent Zdzislaw Sowiński.

Ehe ich zu den Ergebnissen meiner pathologisch-anatomischen Untersuchungen über Pityriasis rubra pilaris übergehe, will ich kurz andeuten, welches hervorragendes Interesse und welche besondere Stellung dieser Affektion in der Pathologie der Hautkrankheiten gebührt. Obgleich diese Krankheitsform, allerdings nur ganz ungenau, zum ersten Male von Devergie im Jahre 1857 unter dem Namen "Pityriasis pilaris" beschrieben worden ist, so wurde sie dennoch viele Jahre hindurch mit der Psoriasis verwechselt, und erst durch die viel später veröffentlichten Untersuchungen Besniers (1) wurde dieses Leiden als selbständige Krankheitsform festgestellt.

Im Jahre 1862 hat Hebra unter dem Namen „Lichen ruber" zum ersten Male eine seltene Hautkrankheit beschrieben, im Jahre 1869 aber hat Erasmus Wilson eine andere Krankheitsform als "Lichen planus" bezeichnet.

Kaposi kam im Jahre 1877 zu dem Schlusse, daß die von $\mathrm{Hebra}$ und von Wils on beschriebenen Krankheiten und Varietäten ein- und derselben pathologischen Form sind und schlug infolgedessen vor, die Form von $\mathrm{Hebra}$ als "Licheu ruber acuminatus", die von Wils o n aber als "Lichen ruber planus" zu bezeichnen. Diese letztere Form rief unter den Dermatologen keine Meinungsverschiedenheient hervor und wurde 
sofort von allen anerkannt. Was jedoch den "Lichen ruber" Hebras oder den "Lichen ruber acuminatus" von Kaposi anbetrifft, so ist noch bis auf unsere Tage die Frage dieser Krankheitsform nicht endgültig aufgeklärt. Auf dem internationalen Kongreß zu Paris im Jahre 1889, wo der über die Benennung dieser Krankheit entstandene Streit zur Rede kam und auch mehrere Fälle derselben demonstriert wurden, konstatierte man, daß der "Lichen ruber acuminatus" der deutschen Verfasser nichts anderes ist, als wie die "Pityriasis rubra pilaris" von Devergie, Riebaud, Besnier (2). In demselben Sinne wurde die strittige Frage auf dem XII. internationalen Kongreß zu Moskau, wo bei ein und demselben Patienten von französischen Ärzten, mit Prof. Gaucher an der Spitze, typische Pityriasis rubra pilaris, von Kaposi, Schwimmer, Behrend u. a. aber Lichen ruber acuminatus $K$ aposi [Lichen ruber $\mathrm{Hebra}(3)]$ festgestellt wurde, entschieden. Die französische Schule erkennt also den Lichen ruber acuminatus nicht als selbständige Krankheit an, sondern hält ihn für eine Varietät einer als "Lichen ruber" zu bezeichnenden Krankheitsform; je nachdem, ob bei dieser letzteren kegelförmige oder platte primäre morphologische Elemente vorwalten, werden der $\mathrm{Be}$ nennung die entsprechenden Adjektiva "acuminatus" oder "planus" hinzugefügt.

Was hauptsächlich gegen die Identität des Lichen ruber von $\mathrm{Hebra}$ und der Pityriasis rubra pilaris von DevergieBesnier sprach, war der Umstand, daß die ersten 14 Fälle $\mathrm{Hebras}$ einen letalen Verlauf nahmen, während die Pityriasis rubra plaris nicht unmittelbar tödlich ist, weshalb diese letztere Krankheitsform, deren klinisches Bild zudem undeutlich war, von einigen Autoren nicht mehr beachtet wurde. Bekanntlich aber endeten alle später von $\mathrm{Hebra}$ und $\mathrm{Kaposi}$ beobachteten Fälle, 42 an der Zahl, mit vollkommener Genesung. Weiter sagt $\mathrm{H} \mathrm{e} \mathrm{bra} \mathrm{(4)} \mathrm{sogar,} \mathrm{daß} \mathrm{er} \mathrm{weder} \mathrm{unter} \mathrm{seinen}$ Beobachtungen, noch unter denen anderer Ärzte irgend einen Fall kennt, in dem der einmal geheilte Lichen ruber zu einem Rückfalle Anlaß gegeben hätte. An anderer Stelle sagt er, daf der Fall von Rosner, welchen Biesiadecki angibt, wahrscheinlich $\mathrm{zu}$ denjenigen gehört, in welchen noch keine 
vollständige Genesung eingetreten war. Es beruht also der Einwand, welchen viele Autoren gegen die Form Hebras gemacht haben, nicht auf fester Grundlage.

$\mathrm{H}$ e b r a hat den Lichen ruber genau beschrieben und trotzdem, daß seit seiner ersten Beschreibung dieser Krankheitsform viele Jahre verstrichen sind, finden wir in der Literatur nichts Neues über sie. Die Frage von der Ätiologie derselben bildet einen der dunklen Punkte der Pathologie der Hautkrankheiten. Die von $\mathrm{Hebra}$ vorgeschriebene Therapie ist nur sehr wenig vorwärts geschritten. Am Schlusse des diese Krankheitsform behandelnden Kapitels sagt $\mathrm{Heb} \mathbf{r a}$, daß das Arsen immer noch als wesentlichstes Heilmittel gegen Lichen ruber anzuerkennen ist. Seit jener Zeit sind 45 Jahre verflossen, und wir können wohl kaum zu diesen Worten noch viel Neues hinzufügen. Um das eben Gesagte zu bekräftigen, soll hier in kurzen Worten das von $\mathrm{Hebra}$ beschriebene Krankheitsbild wiedergegeben werden.

In seiner noch bis heute klassischen Beschreibung teilt $\mathrm{Heb}$ a den Lichen ruber (Pityriasis rubra pilaris Devergi eBesnier) in 3 Perioden ein. Die ersten 2 Perioden bieten nichts Besonderes. Es sind dieses diejenigen Formen, welche man auf der Höhe des Krankheitsprozesses beobachten kann und welche sich nur durch den Grad und den Umfang der Affektion von einander unterscheiden, wobei das als Knötchen auftretende primäre Element sowohl in der ersten, als auch in der zweiten Periode festgestellt werden kann. In die dritte Periode reiht $\mathrm{Hebra}$ diejenigen Fälle ein, in welchen infolge ausgiebiger Knötchenbildung sich die Haut schließlich rötet und verdickt, glatt oder gleichsam mit feinen Papillen besät ist (Chagrinlederbaut); die Epidermis, namentlich ihre äußersten Schichten, liegt in Form von dünnen, grauweißen, leicht abfallenden Schüppchen da; von Knötchenbildung ist keine Spur mehr zu finden. Alteration der Nägel, behinderte Beweglichkeit der Gelenke, Rhagaden usw. sind auch in dieser Periode zu beobachten. Natürlich bieten Kranke in dieser Periode, wo das primäre morphologische Element bereits fehlt, bedeutende Schwierigkeiten in Bezug auf die Diagnose und es ist nicht zu verwundern, wenn ein und derselbe Patient, welcher zu 
verschiedenen Zeiten an verschiedene Kliniken kommt, in ihnen unter verschiedenen Namen behandelt wird; am häufigsten bezeichnet man die Krankheit als "Psoriasis" oder "Parapsoriasis".

Ganz abgesehen von dem Wirrwarr, welcher in der Pathologie durch Einbürgerung verschiedener Benennungen für ein und dieselbe Krankheit entsteht, hat die uns interessierende Krankheit auch rom anatomischen Standpunkte aus mit dem "Lichen ruber" der französischen Autoren durchaus nichts gemein. Während beim "Lichen ruber" die akut entzündlichen Knötchengebilde aus der Tiefe hervorgehen und die Hornschicht est sekundär affizieren, gehören die primären Knötchen der Pityriasis rubra pilaris zu den Veränderungen der Hornschicht, welche von den Haarbalgfollikeln ausgehen und entwickeln sich in diesem Falle entzündliche Veränderungen in deren Umgebung erst sekundär ( $\mathrm{Jacquet}$ ).

Die Pityriasis rubra pilaris stellt eine überaus seltene Hautkrankheit dar, noch seltener sind die rom anatomischen Standpunkte aus beschriebenen Fälle dieser Krankheit. Dieser Umstand bewog mich, 3 diesbezügliche Fälle aus der Klinik des Herrn Prof. T. P. Pawlow genauer zu beobachten und sie vor allem einer pathologisch-anatomischen Untersuchung zu unterwerfen, wobei ich auch die neuesten Ergebnisse über die Entstehung zelliger Elemente der entzündlichen Neubildung mit in Betracht zog. Die gesamte Gruppe der Keratosen und Parakeratosen bietet ja in gewissen Stadien, ganz gleich ob sie von lokalen Bedingungeu oder anderen Ursachen abhängen, rom klinischen Standpunkte aus gar kein Interesse und deshalb ist es nicht zu verwundern, daß noch bis zum heutigen Tage der Streit über verschiedene Benennungen nicht abgeschlossen ist und daß einige Verfasser, wie z. B. T e n n e s on (2), die Pityriasis rubra pilaris als Psoriasis anzusehen geneigt sind.

Da dieses Exanthem nicht näßt, keinen Juckreiz und überhaupt keine Beschwerden verursacht, sodann in seinen Anfangsstadien nur unbedeutende Flächen einnimmt und zudem hauptsächlich die ärmsten Bevölkerungsklassen von ihm betroffen werden, so kommt es in seinen Initialstadien nur selten zur Beobachtung. 
Auf Grund der oben angeführten Erwägungen halte ich es für überaus wichtig festzustellen, ob in der Tat die einander ähnlichen Krankheiten "Lichen ruber", "Psoriasis" und „Pityriasis rubra pilaris" vom anatomischen Standpunkte aus verwandte Erkrankungen oder nur Varietäten ein und derselben Krankheit oder schließlich ganz verschiedene Krankheiten darstellen. Diese Frage kann nur durch genaues Studium des spärlichen Materiales, welches in unsere Hände gelangt, entschieden werden.

Wie bereits erwähnt, konnte ich 3 Fälle dieser Art beobachten; ehe ich jedoch zu den Ergebnissen meiner Untersuchungen übergehe, will ich die Krankengeschichten kurz wiedergeben, zumal als einer der zu beschreibenden Fälle seinem klinischen Bilde nach ganz besonders interessant war:

1. (Eigener Fall.) E., 32 Jahre alter Bauer, Mann von mittlerem Wuchse, normalem Körperbau und Ernährungszustande, sowie gut entwickeltem Knochen- und Muskelsystem; im Dezember 1906 in die Klinik aufgenommen. Schleimhäute normal gefärbt. In den inneren Organen keine Veränderungen. Der Harn enthält keine pathologischen Elemente. Patellarreflexe, sowie Schleimbautreflexe gesteigert. Gesichtsfeld des linken Auges etwas eingeengt $(\mathrm{V}=20 / \mathrm{XXX})$. Am Halse, am Rumpf sowohl vorn, als auch hinten, an Beuge- und Streckfächen der Arme und am Gesäß, von wo aus das Exanthem auch auf die unteren Extremitäten übergreift, stellt die Haut des Patienten eine trockene, schuppende und gerötete feinhöckerige Oberfäche dar, wobei die Unebenheiten an diesen Stellen parallel verlaufende, hervorspringende Wülste bilden. Am Halse sieht man in diesen Wülsten außerdern stellenweise noch vereinzelte Hornknötchen. Die Schuppenbildung ist ausgiebig, kleieartig. Die behaarte Kopfhaut weist auch kleieartige Schuppenbildung auf glatter, geröteter Oberfläche auf; die Haare fühlen sich trocken an; sie sind hart und stehen vereinzelt da; Alopecie der Scheitelgegend. Die Gesichtshaut zeigt fettigen Glanz, ist gerötet, stellenweise gewahrt man auch hier Schuppenbildung, so daß das Gesicht des Kranken wie gespannt aussieht. Untere Augenlider zum Teil ektropisch. An den äuBeren Augenwinkeln - kleieartige Schuppenbildung. Die Wimpern an den äußeren Teilen der Augenlider in spärlicher Anzahl verstreut. An den Handfächen waren weder Knötchen noch Rötung zu gewahren, wohl aber ausgiebige Verhornung und Schuppenbildung, wobei die normalen Hautfalten dentlicher ausgeprägt waren als wie gewöhnlich. Dieselben Veränderungen, jedoch in viel stärkerem Grade, waren anch an den Fußsohlen zu vermerken. Die Fingernägel sind am freien Ende dentlich verdickt; unter ihnen sieht man Ablagerungen von Hornsubstanz. Außerdem zeigen die Nägel an ihrem freien Rande deutliche I,ängsstrichelung und 
Gelbfärbung. Dieselben Veränderungen finden sich auch an den Fußnägeln.

Durch Nachfrage konnte festgestellt werden, daß Patient an seiner Krankheit schon 17 Jahre leidet. Von Interesse ist der Umstand, daß sich bei ihm Remissionen der Krankheit nicht im Sommer, wie das häufiger zur Beobachtung kommt, sondern im Winter einstellten. Seit zwei Jahren besteht das Exanthem ohne Unterbrechung. Vor diesem Zeitraume waren im Laufe von 7 Jahren keine Krankheitssymptome zu beobachten gewesen: die Haut des Patienten war in diesem Zeitraume durchaus normal. Der Vater des Pat. ist am Leben und erfreut sich einer guten Gesundheit. Pat. ist verheiratet und hat zwei Kinder gehabt, die jedoch beide gegen Ende ihres ersten Lebensjahres gestorben sind. Über den Beginn seiner Krankheit erzählt er folgendes: Sein Vater heiratete zum zweiten Male eine Witwe, die bereits erwachsene Söhne hatte; als einst ein Streit $z$ wischen den Stiefsöhnen und dem Vater entstand, verjagten die ersteren sowohl ihn, als auch unseren Patienten aus dem Hause und drohten noch mit weiteren Gewaltsamkeiten. All diese Unannehmlichkeiten wirkten sehr stark auf unseren Patienten ein, und nach Verlauf von 8 Monaten zeigte sich bei ihm das Exanthem am Halse, um sich bald auch auf den Rumpf fortzusetzen. Übrigens hatte noch 3-4 Monate vor diesem Ereignis ein anderes Exanthem, welches akut aufgetreten und bald wieder verschwunden war, den gan zen Körper des Patienten bedeckt. Gegenwärtig klagt Patient über ein unangenehmes Gefühl von Trockenheit und Spannung der Haut. Schweißsekretion und Juckreiz fehlen ganz. Alkohol- und Tabakmißbrauch leugnet Patient vollständig; auch an Syphilis hat er nicht gelitten.

Die anatomischen Befunde berechtigen zu dem Schlusse, daß wir es im gegebenen Falle mit einer chronischen Hautkrankheit, welche zeitweise ganz verschwindet und das Allgemeinbefinden des Patienten augenscheinlich gar nicht beeinflußt, zu tun haben. Faßt man die Befunde der Anamnese und der klinischen Untersuchung zusammen, so muß man unwillkürlich an die chronischen Exantheme, welche durch Schuppenbildung charakterisiert sind, denken. Von diesen kennen wir die Psoriasis, den Lichen ruber, die Ichthyosis und die Pityriasis rubra pilaris. Während die primären Elemente der beiden ersteren Erkrankungen auf geröteter, verändeter Basis verstreut sind, kommen die Elemente der Pityriasis auch auf ganz normaler Haut vor. Bevor man sich für die eine oder die andere klinische Form entscheidet, muß man erst die typischen, primären, morphologischen Elemente, aus denen sich die betreffende Krankheitsform herausgebildet hat, ausfindig 
machen. Bei aufmerksamer Betrachtung der Handrücken, des unteren Vorderarmdrittels und der Dorsalfläche der Fingerphalangen, sowie der Innenfläche der oberen Schenkelteile konnte eine Reihe kleiner Hornsubstanzknötchen, welche auf ganz normaler Haut vereinzelt verstreut waren und der Hautoberfläche ein höckeriges Aussehen verliehen, leicht erkannt werden. Stellenweise war das Zentrum dieser Knötchen mit einem abgebrochenen Haar durchsetzt und dann sah man an dieser Stelle ein schwarzes Pünktchen. Wenn ich nun also die Ergebnisse der klinischen Untersuchung in Betracht zog und andere ähnliche Frkrankungen ausschloß, so sah ich mich berechtigt, im gegebenen Falle eine Pityriasis rubra pilaris Devergie-Besnier anzunehmen. Es sei außerdem bemerkt, daß ein Teil der primären Knötchen an den Handrücken auftrat, als Patient sich bereits auf der Klinik in meiner Beobachtung befand. Diese eben erst in ihrer Entwicklung begriffenen primären morphologischen Elemente dienten mir im gegebenen Faile als Material für meine pathologisch anatomischen Untersuchungen.

2. A. G., 39 Jahre alter Bauer, befand sich zum ersten Male in November 1899, zum zweiten Male im Dezember 1904 und zum dritten Male vom 20. Oktober 1905 an auf der Klinik in Behandlung. Da meine anatomischen Untersuchungen sich auf das Jahr 1905 beziehen, so gebe ich hier nur das klinische Bild dieser letzten Periode.

Die Krankheitsdauer beträgt ca. 9 Jahre. In Anfang verspürte Patient ein leichtes Jucken im Genick, dann rötete sich die Haut in dieser Gegend und trat das Exanthem auf. Vordem war Patient stets gesund gewesen und litt nur in demselben Jahre, wo er vom Exanthem befallen wurde, häufig an Schüttelfrost, auf den starke Gliederschmerzen folgten. Vor der Krankheit mußte Patient in feuchtem Boden arbeiten, da er an Kanalarbeiten teilnabm. Vater und Mutter des Patienten sind gesund; in der Familie sind weder Syphilis, noch Tuberkulose vorgekommen. Patient ist verheiratet und Vater eines Kindes.

Stat. praes: Mann von mittlerem Wuchs, starkem Körperbau, gutem Ernährungszustande. Kniereflexe abgeschwächt. Schleimhäute normal gefärbt. An der behaarten Kopfhaut kleieartige Schuppenbilduny. An den Ohrmuscheln, über den Augenbrauen, an der Nasenspitze, den Wangen und dem Kinn bedeutende Auflagerung von schmutziggranen Schüppchen, welche der Hautoberfläche fest anhaften. Am Gesicht kleieartige Schuppenbildung; Gesichtshaut derb und etwas raub. Die ganze Körperoberfläche rot und runzelig; überall ausgiebige Schuppenbildung, welche besonders stark an den Streckfächen der Arme und in der Lenden- 
gegend ausgeprägt ist. Die Körperoberfläche erinnert an Chagrinleder und fühlt sich rauh und trocken an. Am Penis und am Skrotam ist die Haut etwas gerötet, die Innenfläche der Oberschenkel ist fast normal. Im Subskapulargebiet, an den Streckflächen der Unterarme, sowie im Gebiet der Mm. glutaei und an den oberen Dritteln der Oberschenkel erscheint die Haut zum Teil normal, zum Teil aber, namentlich in der Subskapulargegend, mit stecknadelkopf- bis hanfkorngroßen kegelförmigen Knötchen besät; an der Spitze dieser Knötchen gewahrt man Häufchen verhornter Schüppchen. Diese Gebiete sind vollkommen symmetrisch gelagert. An den Räckenflächen der Hände ist die Haut trocken und rauh, schmutziggrau verfärbt und mit einer Reihe kleiner spitzer vereinzelt dastehender Hornsubstanzknötchen besät. Derartige Knötchen finden sich auch an den unteren Unterarmteilen, sowie an den Handfingern. Stellenweise sieht man im Zentrum derselben ein abgebrochenes Haar. Die Haut an den Handfächen ist stark verhornt, stellenweise in der Richtung der natürlichen Hautfalten von tiefen Einrissen durchsetzt. Die Hautfalten sind bedeutend stärker ausgeprägt, als wie im normalen Zustande. Die an ihrem freien Rande stark verdickten Nägel weisen schnutziggelbe Färbung und deutliche Längsstrichelung auf; unter ihrem freien Rande befinden sich Ansammlungen von Hornsubstanz. Dieselben Veränderungen finden sich auch an den Fußsohlen, nur sind hier Hautverhornung, Rhagaden, Veränderungen des freien Nagelrandes und Ablagerungen von Hornsubstanz stärker ausgeprägt. Der linke Unterschenkel zeigt gar keine Schuppenbildung und ist normal gefärbt. Sobald sich Patient entkleidete, so sammelte sich um ihn herum am Boden eine Menge Hautschüppchen an, so daß der Boden wie mit Mehl bestreut schien. Zum Beweis, daß Patient sich durchaus gesund fühlte, mag erwähnt werden, daß er aus dem Gouvernement Witebsk zu Fuß gekommen war, um an der Klinik Aufnahme zu finden.

3. J. B., 40 Jahre alter Bauer, war $7 \mathrm{mal}$ an der Klinik in Behandlung, zum ersten Male vom 20. März 1902 an, dann in den Jahren 1903, 1904 und 1905. Kann nicht genau angeben, wann seine Krankheit begonnen hat; jedenfalls ist er schon mehrere Jahre krank gewesen.

Stat. praes: Mann von hohem Wuchs, gutem Ernährungszustande, mit normal entwickeltem Knochen- und Muskelsystem. Normale Färbung der Schleimhäute. Gesichtshaut blaurot, glänzend, gleichsam mit Lack überzogen und gespannt. Behaarung dicht, die Haare fühlen sich trocken an. Die ganze Körperhaut, sowie die Haut an den Extremitäten ist hellrot verfärbt, atrophisch, runzelig, reichlich mit silberglänzenden Schüppchen bedeckt, welche stellenweise, namentlich am Rücken, ziemlich mächtige Ablagerungen bilden; nimmt man diese Schuppen ab, so gewahrt man die rote, zum Teil atrophische, nirgends nässende Haut. Stellenweise, namentlich am Bauche, ist die Rotfärbung der Haut keine fortlaufende, und hier kann man kleine, hanfkorngroße einzeln stehende rote Knötchen, welche ihrer Lage nach den Follikeln entsprechen, ausfindig machen. Die Spitze dieser Knötchen ist mit weißen Schüppchen bedeckt; zwischen 
den ziemlich dicht stehenden Knötchen ist die Haut ihrer Farbe nach vollkommen normal. Dieselben Veränderungen sind an den unteren 'Teilen der Unterarme zu vermerken.

Wie bereits oben erwähnt, teilt Hebra den Verlauf seines "Lichen ruber" ("Pityriasis rubra pilaris", D evergie) in 3 Perioden ein. Oben ist auch hervorgehoben worden, daß diese Krankheit, da sie die Kranken wenig beunruhigt, selten in ihren Anfangsstadien den Ärzten zu Gesicht kommt und erst, wenn sie große Körpergebiete, namentlich die Gesichtshaut, eingenommen hat, die Patienten aus rein ästhetischen Rücksichten zwingt, sich an einen Arzt zu wenden, Es versteht sich von selbst, daß man sich das anatomische Bild dieses Anfangsstadiums nur mit großen Schwierigkeiten verschaffen kann, daß dieses jedoch zugleich höchst erwünscht ist. Auf diese Weise hätten wir die Möglichkeit, die strittige Frage zu lösen und könnten wir zugleich Einwände über die primäre Lokalisation des Prozesses beseitigen. Oie beiden zuerst beschriebenen Fälle bieten das klinische Bild einer vollkommen ausgebildeten "Pityriasis rubra pilaris". Was jedoch den 3. Fall anbetrifft, so war es sehr schwierig, ihn in dieser Periode direkt zu der "Pityriasis rubra pilaris" zu beziehen, da nur sehr wenige für diese Krankheit charakteristische Merkmale zu verzeichnen waren. Er stellt nämlich das dritte Krankheitsstadium dar, und natürlicher Weise bietet diese Form in diagnostischer Beziehung bedeutendes Interesse. In diesen Fällen könnte nur das anatomische Bild die Frage entscheiden; leider beziehen sich die anatomischen Befunde, welche vou den Autoren erwähnt werden, ausschließlich auf die 2. Krankheitsperiode. Ich halte deshalb die von mir beschriebenen Fälle für beachtenswert und zwar sowohl aus dem Grunde, weil überhaupt diese Hautkrankheit zu den Seltenheiten gehört, als auch weil ich hier das histologische Bild verschiedener Krankheitsstadien ausmalen kann.

Zur Anfertigung anatomischer Schnitte dienten mir Hautstücke, welche nach vorhergehender Kokaininjektion an folgenden Stellen excidiert worden waren. In Fall 1 wurde ein Stückchen, welches die charakteristischen primären morphologischen Elemente enthielt, an der Rückfläche der linken Hand 
ausgeschnitten; die anderen Stückchen stammten von symmetrischen Gebieten der Unterschenkel. Dieses Gebiet war nicht nur im gegebenen Moment vollkommen normal, sondern war auch früher nie Sitz des Krankheitsprozesses gewesen. Ich verfuhr so, um Vergleiche, über deren Ergebnisse weiter unten die Rede sein soll, anstellen zu können. Das in Fall 2 aus der Unterarmhaut ausgeschnittene Hautstückchen enthielt etwa stecknadelkopfgroße morphologische Elemente und zeigte fast normale Körperfarbe ; einige Knötchen waren mit zarten Hautschüppchen bedeckt. In Fall 3 wurde ein Hautstückchen aus dem Interskapulargebiet excidiert; an seiner Oberfläche sah man dicht neben einander stehende, kleine, rote Elemente von unregelmäBiger Form und ohne merkbare Schuppenbildung; da die einzelnen Elemente nicht von einander getrennt waren, so stellte das ausgeschnittene Stückchen gleichsam eine fortlaufende Affektion, welche der Haut ein chagrinlederartiges Aussehen verlieh, dar und konnte man überhaupt das Vorhandensein vereinzelter Elemente bestreiten. ${ }^{1}$ )

In Fall 1 wurde das Stückchen in Alkohol, sowie in konzentrierter Sublimatlösung, welche aus physiologischer Kochsalzlösung mit Zusatz von einigen Tropfen Ac. aceticum glaciale angefertigt worden war, gehärtet. Dieses ist die ein wenig modifizierte Methode von Sabouraud, welche aus folgenden Manipulationen besteht: 1. der Schnitt wird auf eine Stunde in die oben erwähnte Sublimatlösung mit Essigsäurezusatz gelegt, hierauf kommt er auf zwei Stunden in Aceton, welches leicht mit Jodtinktur angefärbt ist, wobei in dem Falle, daß die Lösung sich entfärbt, wiederum Jodtinktur bis zu einer Färbung, welche an Madeirawein erinnert, hinzugefügt werden muß; 2. der Schnitt wird zweimal im Laufe von je 2 Stunden in reinem Aceton ausgewaschen; 3. er verbleibt im Laufe von 8-12 Stunden in einer konzentrierten Lösung von Paraffin in

1) Zu Fall 2 und 3 benutzte ich bereits eingebettete Stückchen, welche mir von meinem Kollegen, Herrn Dr. W. J. Terebinsky, dem ich an dieser Stelle meinen verbindlichsten Dank ausdrücke, zur Verfügung gestellt worden waren. Herzlichen Dank bin ich auch Herrn Dr. N. W. Morosow schuldig, welcher in Fall I die Exzision der Hantstückchen vornahm. 
Äther bei $37^{\circ} \mathrm{C}$. und wird sodann in Paraffin eingebettet. Ich härtete die Stückchen auch in $Z$ enkerscher Lösung. In Fall 2 und 3 wurden die Hautstïckchen in Alkokol, Sublimat und Zenkerscher Flüssigkeitfixiert und in Zelloidin und Paraffin eingebettet.

Von Farben verwandte ich $D$ e la fi eld sches Hämatoxvlin, Eosin, Safranin, Lichtgrün; außerdem wandte ich die v. Giesonsche Methode, die Methode von Gram nebst darauffolgender Tinktion mit Orth schem Karmin und die Färbung mit polychromem Methylenblau nach Unna an. Da ich in den Details den allgemein anerkannten Vorschriften folgte, so halte ich eine Beschreibung derselben hier für überflüssig.

Ehe zur Beschreibung der anatomischen Veränderungen, welche in den von mir untersuchten Fällen gefunden wurden, übergehe, möcnte ich noch der Theorien, welche gegenwärtig über die Entstehung der zelligen Elemente der entzündlichen Neubildung herrschen, erwähnen.

Derartige Theorien sind uns zwei bekannt. Die eine von ihnen war bis vor kurzem die einzig anerkannte. Dieses ist die histiogene Theorie, deren Anhänger annahmen, daß die Elemente der entzündlichen Neubildung das Lrgebnis der Vermehrung präformierter Bindegewebselemente sind. Als Repräsentant dieser. Theorie ist unter den Dermatologen Unna anzusehen.

In letzter Zeit haben sich die Ansichten über diese Frage bedeutend verändert und schließlich ist eine neue Lehre, die sog. hämatogene Theorie entstanden, nach welcher die Zellen der entzündlichen Neubildung aus mononukleären Blutelementen, welche aus der Blutbahn emigriert sind, entstehen. Die umfangreiche Gruppe der Leukocyten besteht aus sehr verschiedenartigen Zellformen und erst die bahnbrechenden Untersuchungen $\mathrm{Ehrlichs}(5)$ haben eine rationelle Klassifikation, welcher einerseits die Beschaffenheit des Kernes, andererseits aber die Beschaffenheit des Protoplasmas zu Grunde gelegt wurde, geschaffen. Auf diese Weise unterscheidet man also Leukocyten mit rundem oder etwas unregelmäßig geformtem Kerne und basophilem Protoplasma, welches in frischem Zustande keine färbbaren Körncben enthält. Diese Zellen werden als Lympho- 
cyten bezeichnet [Pappenheim (6), A. Maximow u. a.]. Andererseits existiert eine Gruppe von Leukocyten mit verschiedenartig geformtem Kern und mit deutlich wahrnehmbarer Körnelung des Protoplasmas. welche eine typische Affinität zu verschiedenen Anilinfarben offenbart. Diese Zellen tragen nach Pappenheim (7) den Namen Granulocyten oder Leukocyten im speziellen Sinne. Die verschiedenartige Affinität der Körnchen dieser Leukocyten zu verschiedenen Anilinfarben, welche von $\mathrm{E} \mathrm{h} \mathrm{r-}$ li $\mathrm{ch}$ entdeckt worden ist, hat als Grundlage für die Einteilung der Leukocyten in 3 Arten gedient; es sind dieses: 1. Leukocyten mit spezieller Granulation; 2. solche mit eosinophiler oder acidophiler Granulation und 3. Leukocyten mit basophiler Granulation, sog. Mastzellen.

Um wieder auf die Lymphocyten zurückzukommen, darf nicht unerwähnt bleiben, daß ihre Fähigkeit, aktive Bewegungen auszuüben und also auch aus den Blutgefäßen zu emigrieren, lange Zeit über ganz und gar geleugnet wurde. Erst in letzter Zeit hat Prof. A. Maximow (8) diese Frage auf experimentellem Wege zu untersuchen getrachtet und es ist ihm gelungen, durch direkte Experimente zu beweisen, daß sowohl große als auch kleine Lymphocyten zweifellos eine sehr rege Mobilität besitzen, und daß sie bei Entzündungen ebenso aus den Blutgefäßen auswandern, wie die polymorphkernigen Leukocyten. Die Ergebnisse dieser Untersuchungen bestätigten die Ansicht J. J. Metschnikows (8), welche er schon früher auf Grund der Befunde der vergleichenden Pathologie ausgesprochen hatte.

Indem ich dem Studium der pathologisch-anatomischen Veränderungen in den von mir untersuchten Fällen diese neue Anschauung über den Ursprung der zelligen Elemente zu Grunde lege, will ich der Beschreibung einiger Zellformen, welche weiter unten bei der Wiedergabe des anatomischen Bildes zu erwähnen sein werden, hier einige Zeilen widmen.

Die „Polyblasten" stellen eine Varietät der Lymphocyten, welche verschiedene Form und Größe zeigt, dar. Sie sind entweder aus Lymphocyten, welche die Blutgefäße verlassen und sich weiter entwickelt haben, oder aus fixen Bindegewebszellen, welche ihre Entstehung gleichfalls Leukocyten, die jedoch be- 
reits in der Embryonalperiode des Organismus die Blutgefäße verlassen haben, verdanken, entstanden. Die Zellen der Polyblasten besitzen verschiedene Größe und es finden sich zwischen ihnen sämtliche Übergangsformen zwischen mononukleären Rundzellen einerseits und sogenannten zwei- bis dreikernigen Epitheloidzellen andererseits. Was jedoch den Kern anbetrifft, so behält er im allgemeinen die Struktur des Lymphocytenkernes bei und färbt er sich stets bedeutend intensiver, als wie das Protoplasma; die Chromatinsubstanz ist in ihnen dichter verteilt, als wie in anderen Zellen von der gleichen Größe. Die Grundform des Kernes ist die kugelige, obgleich auch einige Abweichungen von dieser gewöhnlichen Form nicht ausgeschlossen sind. Liegt der Kern im Zentrum der Zelle, so ist er meist rund, verschiebt er sich jedoch zu dem Rande des Zelleibes, so ist er entweder rund oder mehr oder weniger verlängert, zuweilen sogar bogenförmig. Die Größe des Kerns ist eine verschiedenartige und sie kann die Größe des Lymphocytenkerns um ein Vielfaches übertreften. In dem Kerne gewahrt man $1-2$ große Nukleoli, welche meist sehr deutlich zu unterscheiden sind. Der Kern ist scharf umrissen. Das Protoplasma tritt deutlich hervor, wobei in alten Zellen in ihm gleichfalls deutliche Granulation bemerkbar wird. Die Zellen besitzen entweder runde oder ovale, zuweilen auch polygonale Form, jedoch sind lange, lanzenförmige Protoplasmafortsätze an diesen Zellen nicht zu gewahren. Aus Beobachtungen A. Maximows geht hervor, daß diese Zellen in Plasmazellen, welche den Grundstock der Granulome bilden, übergehen.

Die "Fibroblasten“ sind Bindegewebszellen von ganz verschiedener Größe und Form. Auch hier unterscheidet man Kern und Zellprotoplasma. Gewöhnlich besitzt der Kern eine verlängerte Eiform. In einigen Fällen ist der Kern biskuitförmig, wobei man auch Teilungsfiguren in ihm gewahrt. Die Chromatinmenge der Kerne ist ihrer Größe umgekehrt proportional und während in kleinen Kernen das Chromatinnetz deutlich hervortritt, ist die Chromatinmenge in großen Kernen eine unbedeutende. Außerdem treten in großen Kernen auf blassen Untergrunde zwischen deutlichen Granulationen 1-2 Körnchen, 
welche als Nukleoli bezeichnet werden, hervor. Jedenfalls färbt sich hier der Kern weniger scharf, als wie bei den Lymphocyten; jedoch treten seine Umrisse ziemlich deutlich hervor. Die Fibroblasten sind mit Fortsätzen versehen und können je nach der Form und Menge dieser Fortsätze das Aussehen einer Spindel oder eines Sternes gewinnen. Im Vergleich zur Größe des Kernes ist die Protoplasmamenge eine verschwindende, zuweilen kann das Protoplasma überhaupt nicht nachgewiesen werden, und dann scheint der Kern mit den Fortsätzen eng verbunden zu sein. Das Protoplasma zeigt netzartige Struktur. Als Unterscheidungsmerkmale von großen Polyblasten dienen folgende: die langen Protoplasmafortsätze der Fibroblasten, die blassere Färbung ihrer Kerne, in einigen Fällen aber auch der Umstand, daß das Protoplasma bei Fibroblasten fast ganz fehlt.

Die "Mastzellen" unterscheiden sich bei Bearbeitung des Präparates mit Unnas polychromem Methylenblau mit darauffolgender Entfärbung in Glyzerinäthergemisch deutlich von den übrigen Zellenformen. Ihre Kerne bieten nichts Besonderes. Im Protoplasma gewahrt man einige vereinzelte grobe Granula, welche sich bei Bearbeitung der Präparate mit polychromem Methylenblau metachromatisch (rot) färben. Die Zellen besitzen sehr verschiedene Form, sie sind bald in die Länge gezogen, bald gekörnt, bald erinnern sie täuschend an Plasmatocyten mit der diesen eigenen Granulation der Fortsätze und nur das Vorhandensein der metachromatischen Granulation widerspricht dieser Ähnlichkeit. Ich erwähne dieses deshalb, weil in einigen Fällen die Ähnlichkeit mit Plasmatocyten in der Tat eine täuschende war.

Indem ich nun zur Beschreibung des anatomischen Bildes in den von mir untersuchten Fällen übergehe, will ich hinzufügen, $d a ß$ ich unten nur die von mir beobachteten Hauptzüge der Umwandlung von Lymphocyten wiedergebe; die übrigen Zellformen sollen weiter behandelt werden.

Vor allem sei die Hornschicht erwähnt. Diese Schicht war in allen von mir untersuchten Fällen stark verdickt; stellenweise drang die Hornsubstanz in die Follikel der Haarbälge ein und lagerte sich gleichsam um sie herum. In der 
Hornschicht kamen in beträchtlicher Menge Zellen mit plattenförmigen Kernen vor, wobei diese Bezirke mit solchen abwechselten, wo derartige Zellen nicht zu finden waren. Weder eigentliche Leukocyten, noch Lymphocyten waren hier zu sehen.

Das Stratum lucidum $\mathrm{Oehls}$ bestand aus mehreren Schichten. Hier waren auch Zellen mit plattenförmigen Kernen zu beobachten und zwar fanden sich derartige Zellen stellenweise in den oberflächlichen Schichten, während die tieferen Schichten kernlos waren.

Das Stratum Malpighi: Die obere Schicht dieses $\mathrm{Ab}$ schnittes, das sog. Stratum granulosum von Langerhans, bestand stellenweise aus drei Reihen von Zellen, stellenweise aber nur aus einer Reihe. Die Zellen waren platt. In ihrem Protoplasma konnte man deutlich zahlreïche Keratohyalikörnchen unterscheiden. Sowohl Kerne als auch Zellen waren scharf umrissen.

Das Stratum spinosum war saftig und mehrschichtig. Polynukleäre Leukocyten kamen gar nicht vor, zuweilen jedoch sah man Zellen mit stabförmigen Kernen. Fast überall trat die brückenartige Verbindung der Epithelzellen untereinander deutlich $z \mathfrak{u}$ Tage.

Sowohl in der Malpighischen Schicht, als auch hauptsächlich in der basilaren, zylindrischen Schicht waren sehr oft Mitosen zu vermerken. Besonders zahlreiche karyokinetische Figuren sah ich in Fall 1 und 2, in Fall 3 waren sie sehr spärlich vertreten. Außerdem fiel in Fall 2 das reichliche, gelbbraune Pigment in der zylindrischen Schicht und zugleich eine ungeheure Menge von Chromatophoren, was an die Struktur der Naevi erinnerte, auf.

Wenn ich nun die anatomischen Veränderungen mit der Epidermis zusammenfasse, so mul3 ich vor allem diejenigen der Hornschicht verzeichnen. Dieselbe war stets verdickt; außerdem füllten Hornmassen die Haarbalgfollikeln aus und erweckten dadurch, daß sie gleichsam ausschließlich in deren Umgebung konzentriert waren, den Anschein, als ob sie von den Follikeln ausgehen. Das Vorhandensein von Zellen mit platten Kernen, welches vornehmlich in der Hornschicht sowie 
im Stratum lucidum zu vermerken ist, beweist, daß es sich hier nicht um eine komplette Verhornung, sondern nur um eine Parakeratose handelt. Diese anatomischen Befunde stimmen mit den klinischen durchaus überein: bei den Patienten kann sogar in Zeitabschnitten, wo sie unter krankhaften Symptomen nicht zu leiden haben, Hautabschuppung stattfinden.

Es muß noch ein Umstand erwähnt werden und zwar die Karyomitose, welche in Fall 1 und 2 zu beobachten war und in Fall 3 fast ganz fehlte. Diese Erscheinungen zeigen von verstärkter Zellproliferation in diesem Gebiete in Fall 1 und 2. Die Ergebnisse der mikroskopischen Untersuchung in Fall 3 ergänzen sein klinisches Bild. Ruft man sich dasselbe in Erinnerung, so erkennt man deutlich, daß dieser Fall nach den ihn begleitenden Erscheinungen zum dritten Krankheitsstadium nach der Klassifikation von $\mathrm{Hebra}$ gehört. In klinischer Hinsicht äußerte sich die Krankheit in diesem Falle hauptsächlich in Hautrötung und -abschuppung, das anatomische Bild aber, welches auf spärliche Karyokinese hinwies, unterstützte diesen klinischen Befund aufs Treffendste.

Als sehr wichtig muß noch hervorgehoben werden, daß Lymphocyten und eigentliche Leukocyten in sämtlichen 3 Fällen vollständig fehlten.

Die Papillarschicht war aufgelockert; sie enthielt viele erweiterte Interstitien. Die Papillen, welche das Knötchen umgaben, waren nebst der sie überziehenden $\mathrm{Malpighi}$ schen Schicht stark vergrößert, namentlich in Fall 2. Das Bindegewebe war reich an unbeweglichen, ruhenden Zellen (Fibroblasten). Die zwischen den Bündeln ron Bindegewebsfasern verlaufenden Gefäße waren erweitert. Iu der Umgebung der Blutgefäße, in den Bindegewebsinterstitien, gewahrte man eine beträchtliche Ansammlung ron zelligen Elementen, deren Hauptmasse aus Lymphocyten bestand; es kamen auch Fibroblasten vor. Auch Mastzellen waren vorhanden, in Fall 2 in ungeheurer Menge, in Fall 1 vereinzelt, in Fall 3 aber gar nicht. Von Plasmazellen war nichts zu vermerken. Was die Polyblasten anbetrifft, so sah man sie vereinzelt; polynukleäre Leukocyten aber fehlten ganz und gar. Es muß noch bemerkt werden, daß in Fall 1 und 2 gekörnte polymorphe Zellen, 
welche stellenweise in die Länge gezogen waren, trockene, ovale Kerne und grobe Pigmentkörnchen im ganzen Leibe aufwiesen, in ungeheurer Menge vorkamen. Diese Granulation sieht bei Färbung mit Unnas polychromem Methylenblau gelbbraun aus.

Das Verhalten der Haare zu den Haarscheiden war stellenweise ein abnormales. Die Haarscheiden nahmen Trichterform an, wobei ihr erweitertes Ende der Ausgangsöffnung und ihr zugespitztes Ende der Tiefe zu gerichtet war; man gewann den Anschein, als ob der Haarbalg aus mehreren ineinander gelegten Trichtern, in deren Zentrum das Haar sich befand, besteht.

Wie bereits erwähnt, untersuchte ich in Fall 1 nicht nur solche Hautbezirke, an deren Oberfläche die primären morphologischen Elemente zu sehen waren, sondern ganz gesunde aus der Unterschenkelhaut. Auch hier beobachtete ich Gefäßdilatation und bedeutende Ansammlung von Zellen, deren Haupt. masse aus Lymphocyten bestand, in ihrer Umgebung.

Fasse ich die Ergebnisse der anatomischen Untersuchungen in den 3 von mir beschriebenen Fällen zusammen, so muß ich hervorhebən, daß die Veränderungen der Hornschicht in allen sehr scharf ausgeprägt waren, ein Umstand, welcher an eine primäre Affektion derselben denken lassen könnte. Eine andere Erwägung führt jedoch zu $f_{\circ}$ lgenden Voraussetzungen. Die Hornschicht besitzt bekanntlich kein selbständiges Blutgefäßsystem, sondern ernährt sich durch Lymphe, welche aus der Papillarschicht in dieselbe durchdringt; deshalb muß man die Ursachen ihrer Ernährungsstörungen in dieser letzteren suchen. Ich beobachtete in sämtlichen Fällen Veränderungen an den Gefäßen der Papillarschicht; dieselben Veränderungen, allerdings wohl in bedeutend schwächerem Grade ausgeprägt, fanden sich auch in entfernten, ganz gesunden Hautbezirken, welche noch nie Sitz des Krankheitsprozesses waren (in Fall 1). Nimmt man als Ursache der uns interessierenden Erkrankung Veränderungen im Nervensystem an, eine Meinung, die bis heute vertreten wird, so kann man sich die trophischen Störungen, dank welchen diejenige Schicht, die im Falle einer Hemmung der regelmäßigen Zellenernährung am wenigsten 
gesichert ist, vor allem leiden muß, sebr gut erklären. Es versteht sich von selbst, daß die Veränderungen der Hornschicht, weil sie stärker ausgesprochen sind, in den Vordergrund treten und hiermit die eigentliche Ursache verdecken. Geht man also von diesen Erwägungen aus, so muß man eher zugeben, daß bei dieser Erkrankung die als Parakeratose sich äußernden Veränderungen der Hornschicht sekundäre sind.

Schon Hebra wies auf die Veränderungen der Gefäße hin und konnte deren Dilatation vermerken. Auf ihn folgt eine ganze Reihe von Autoren, welche auch auf andere Abweichungen von der Norm hinwiesen.

So beobachtete Neumann (10) Wucherung der Epithelzellen und der Zellen der Malpighischen Schicht, reichliche Zellenansammlung in dem Corium und längs den Gefäßen, welche hauptsächlich in den Papillen verlaufen. Besonders hervorgehoben hat Neumann die Ansammlung von Zellen der äußeren Haarscheide am Boden des Haarfollikels, wo die kegelförmigen Fortsätze, die das Haarfollikel vor sich hervorstülpen, bilden. Biesiadecki bemerkt in dieser Frage, daß die von Neumann angegebenen Veränderungen auch bei anderen chronischen Hautkrankheiten, z. B. am Prurigo, der chronischen Dermatitis, dem chronischen Ekzem vorkommen, während man andererseits auch bei dem Lichen ruber Hebra (Pityriasis rubra pilaris) ein ganz normales Verhalten des im Zentrum des Knötchens befindlichen Haares zu den umgebenden Teilen beobachten kann, weshalb er diese Veränderungen nicht für wesentlich hält.

Wesentliche Bedeutung haben nach Hebras Meinung die Veränderungen, welche Biesiadecki and Kaposi in den Hautpapillen gefunden haben. Die in der Umgebung des Knötchens befindlichen Hautpapillen sind nebst der sie überziehenden Malpigh ischen Schicht vergrößert und mit zelligen Elementen infiltriert. Veränderungen derselben Art konnten auch in dem den Papillen anliegenden Teile der Cutis beobachtet werden. Was jedoch die Papillen anbetrifft, welche im Zentrum des Knötchens gelagert sind, so bieten sie ein geschrumpftes Aussehen. Diese zentralen Papillen entsprechen den Vertiefungen, welche bei klinischer Untersuchung im Zentrum des Knötchens $\mathrm{zu}$ beobachten sind. Nach Untersuchungen von Biesiadecki liegen diese atrophischen Abschnitte stets in der Nähe des Haares und zwar entsprechen sie der Anheftungsstelle der M. erector pili an die obere Schicht der Cutis.

Aus der Literatur, welche die uns interessierende Frage behandelt, sind vor allem die Veröfentlichungen von Richaud (11), Brocq (12), Robinson (13), Taylor (14), Fox (15), Boeck (16), Galewski (17), Hallopeau (18), Audry (19), Unna (20), Neisser (21) und Mourek (22) zu nennen. $\mathrm{Zu}$ den neuesten Veröffentlichungen gehören diejenigen von Lukasiewicz (23), Crocker (24), Havas (25), Lewin (26), Saalfeld (27), Schloemann (28), Török (29), Bredas (30), Róna 
(31), De Amicis (32), Morton (33), Jordan (34), Fox (35), Rosenthal (36), Pollitzer (37), Ravogli (38), Dabreuilh (39), Kalt (40), G. Meschtscherski (41), Palm (42), Leredde (43), Pinkus (44), Du Castel und Kalt (45), Hägel (46), Zieler (47), A lexander (48), Hall, Arthur (49), Blaschko (50), Sokolow (51), Thibierge (52), $\mathrm{Nudelo}$ und Hérisson (53), Vignolo-Lutati (54), Cohn (55) und Breda (56). Hiermit ist die Literatur über Pityriasis rubra pilaris fast erschöpft. Die überwiegende Mehrzahl dieser Veröffentlichungen sind kasuistische Beschreibungen und nur in einigen von lhnen finden sich auch pathologisch-anatomische Untersuchungen.

Um nun zu meinen Fällen zurïckzukommen, muß ich vermerken, daß die Pityriasis rubra pilaris Devergie-Rich a u dBesnier keinen akuten Prozel darstellt, wie der Lichen ruber. Als Beweis hïefür kann der Umstand dienen, daß hier polymorphkernige Leukocyten, welche beim Lichen ruber im Gegenteil sehr zahlreich vertreten sind, ganz fehlen. Ebensowenig handelt es sich bei der Pityriasis rubra pilaris um einen akuten exsudativen Entzündungsprozeß, sondern wir haben es hier mit einer chronisch wirkenden Irritation, welche sich den Blutgefäßen entlang fortpflanzt und zu Ansammlung der oben erwähnten Zellenformen und zu mäßiger Fibroblastenvermehrung führt, zu tun.

Weiter darf nicht unbemerkt bleiben, daß in den von mir beschriebenen Fällen neben polynukleären Leukocyten auch Plasmazellen fehlten; was jedoch die Polyblasten anbetrifft, so kamen sie nur vereinzelt vor. Diese vollkommene Abwesenheit von Plasmazellen und das seltene Vorkommen von Polyblasten zeugen auch gegen den entzündlichen Charakter der uns interessierenden Krankheit, bei welchem der Übergang von Lymphocyten in diese Zellformen unausbleiblich wäre, wie das z. B. bei Syphilis, Lichen ruber usw. zu beobachten ist.

Nimmt man sich das anatomische Bild überhaupt und den Gehalt an verschiedenen Zellformen im Speziellen zur Richtschnur, so muß man die von mir beschriebenen Fälle anders anordnen, als wie ich das bei Beschreibung des kliniBildes getan habe. In Fall 2 fanden sich die meisten Zellenformen, und es mul angenommen werden, dab es dem Höhepunkt der Krankheit entspricht; deshalb muß dieser Fall vom Standpunkte der anatomischen Veränderungen, aus an der Spitze stehen. Weniger Zellformen beobachtete ich in Fall 1 
und ganz arm an diesen war Fall 3. Dieser letzte Fall, welcher aus dem klinischen Bilde nach dem letzten Stadium der Krankkeit (nach der Einteilung von $\mathrm{Hebra}$ ) entspricht, bietet vom anatomischen Standpunkte aus bedeutendes Interesse. Die anatomischen Veränderungen bei Pityriasis rubra pilaris, welche in der Literatur beschrieben werden (Ri e c h a ud, Jacquet u. a.), entsprechen dem Höhepunkt der Krankheit. Hier aber sind einige besondere Erscheinungen zu vermerken, die übrigens einander durchaus nicht widersprechen.

In der Basilarschicht waren nur sehr wenige Mitosen zu beobachten, und obgleich die Gefäße der Papillarschicht erweitert blieben, so waren in ihrer Umgebung weder Zellformen noch Ödem zu beobachten; all dieses bewies, daß das Überleben der zelligen Elemente ein spärliches war und daß dieser Fall zwar nicht ganz dem Endstadium des Krankheitsprozesses entspricht, jedoch jedenfalls sich dem regressiven, atrophischen Stadium nähert. Die in Fall 3, welcher auch vom klinischen Standpunkte aus dem dritten Krankheitsstadium entsprach, zu beobachtenden, anatomischen Veränderungen beweisen uns noch einmal, daß die Pityriasis rubra pilaris keinen akuten Entzündungsprozeß darstellt und daß die Identifizierung dieser Krankheit mit Lichen ruber durch den Vergleich der histologischen Veränderungen nicht bekräftigt werden kann. Das Vorhandensein von Mastzellen und der Umstand, daß die Lymphocyten sich hier nicht in terminale Zellgebilde umwandeln, weisen darauf hin, daß der Prozeß in diesem Stadium gleichsam in seiner weiteren Entwicklung stehen bleibt und nicht in ein Granulom ïbergeht. Diese Erscheinung ist für Prozesse typischer Störung mit Ausgang in Atrophie charakteristisch und diese anatomischen Veränderungen stehen mit dem klinischen Bilde der Erkrankung, bei welcher in der Tat Atrophie zu beobachten ist, in vollkommenem Einklange.

Was die polymorphen, gekörnten Zellen mit trockenen, ovalen Kernen und grober, gelbbrauner Granulation im ganzen Körper, welche ich in Fall 1 und 2 in riesiger Menge zu vermerken hatte, anbetrifft, so sind sie wohl unzweifelhaft Chromatophoren, jedoch muß ich hervorheben, daß sie ihrem Aussehen nach sehr stark an die beim Menschen und bei Säuge- 
tieren beschriebenen Plasmatocyten (R. W.-Zellen) erinnern. Bei dieser Gelegenheit will ich der Voraussetzung Ausdruck geben, daß vielleicht die Chromatophoren Gebilde darstellen, welche mit Clasmatocyten verwandt sind, und daß ein Übergang der einen in die anderen möglich ist. Jedenfalls kann der reichliche Gehalt an Chromatophoren nochmals bestätigen, daß die uns interessierende Krankheit mit chronischer Hyperämie Hand in Hand geht.

Auf Grund der Ergebnisse meiner anatomischen Untersuchungen sehe ich mich also berechtigt, akute Entzündungserscheinungen bei der Pityriasis rubra pilaris zu verneinen und ich schließe mich also der Benennung dieser Krankheit, an welcher die französische Schule festhält, vollkommen an und meine dagegen, daß die Benennung der deutschen Autoren (Lichen ruber acuminatus) den histologischen Veränderungen durchaus nicht entspricht.

\section{Literatur.}

1. Besnier. Pityriasis rubra pilaris. Annales de Dermatol. et de Syphiligr. 1889. p. 253-398-485.

2. Tenneson. Traité clinique de Dermatologie. Paris. 1893. Ga u cher. Traité des maladies de la peau. Paris. 1895. - Pospelow, A. J. Handb. der Hautkrankheiten. Moskau. 1905. - Besnier, Broc q, Jacquet. La pratique dermatologique. Paris.

3. Comptes-rendus du XII Congrès international. Moscou. 1897.

4. Hebra. Handbuch der Hautkrankheiten.

5. Ehrlich und Lazarus. Die Anämie. Spezielle Pathologie $u$. Therapie von Nothnagel. Bd. VIII. I. Teil. 1901.

6. Pappenheim. Erwiderungen auf die kritischen Bemerkungen von Türk. Folia haematologica. 1905.

7. Pappenheim. Atlas der menschlichen Blutzellen. Jena. 1905.

8. Maximow, A. a) Experimentelle Untersuchungen über die entzündliche Neubildung von Bindegewebe. Zieglers Beiträge. Suppl. 5 . Jena. 1802. - b) Ibidem. Bd. XXXIV, XXXV, XXXVIII. - c) Über die Zellformen des lockeren Bindegewebes. Archiv für mikroskop. Anatomie. Bd. LXVII. p. $680-757$. 
9. Metschnik off, J. Leģons sur la pathologie comparée de l'inflammation.

10. Neumann, ref. Hebra, loc. cit.

11. Richaud. Etude sur le Pityriasis pilaris. Ref. Arch. f. Derm. u. Syph. 1906. Bd. LXXIX. p. 273.

12. Brocq. a) Sur le lichen ruber. Ann. de Dermat. et Syphiligr. 1886. p. 389. - b) La question du lichen ruber en Amerique. Annal. de Dermat. et de Syphiligr. 1889. p. 302. - - c) Quelques considerans sur le lichen ruber planus. Journal des praticiens. 1897. Nr. 14.

13. Robinson. a) Lichen ruber and Lichen planus. New-York med. Record. 1883 . $-b$ ) The question of relations hip between lichen planus (Wilson) and lichen ruber ( $\mathrm{Hebra}$ ). Journal of cutaneous and genito-urinary diseases. 1889. Januar-März.

14. Taylor. Lichen ruber as obserwed in America and its distinction from lichen planus. New-York med. Journ. 1889. Januar.

15. Fox. The non identiv of lichen planus and lichen ruber. Ref. Arch. f. Jerm. u. Syph. 1906. Bd. LXXIX. p. 273.

16. Boeck. a) Ein Fall von Pityriasis pilaris. Monatshefte f. prakt. Dermatol. 1889. Bd. VIII. p. 97. - b) Einige Betrachtungen über Lichen ruber in Norwegen. Monatshefte f. prakt. Dermat. 1886.

17. Galewsky. Pityriasis rubra pilaris. Verhandl. der deutsehen dermat. Ges. III. Kongreh. Ergänzungsh, z. Arch. f. Derm. u. Syph. 1892.

18. Hallopeau. a) Sur une variété de lichen de Wilson simulant par places un pityriasis rubra pilaire. Semaine médicale. 1893. - b) Note complementaire sur un eas de lichen de Wilson avec localisation peripilaire (lichen ruber acuminatus) rapports de cette dermatose avec la pityriasis rubra pilaris. Soc. franç. de dermatol. et de syphil. 6 juin. 4 juillet. 1901. - c) Sur une poussée aigné de Pityriasis rubra pilaire avec érythrodermie exfoliatrice généralisée. Ann. de Derm. et de Syph. 1898.

19. Audry. Étude sur le Pityriasis rubra pilaris. Gazette hebdom. de médecine. 1893. janv.

20. Unna. Histopathologie der Hautkrankheiten. 1893.

21. Neisser. a) Zur Stellung der Pityriasis rubra pilaris und des Lichen ruber acuminatus. Verhandl. der Derm. Ges. IV. Kongr. 1894. b) Ein Fall von Pityriasis rubra pilaris. Verhandl. der Breslauer dermat. Vereinigung. Arch. f. Dermat. u. Syph. 1900. Bd. LIII. p. 389.

22. Mourek, Heinrich. Zur Frage der Pityriasis rubra pilaris. Arch. f. Dermat. u. Syph. 1895. Bd. XXXIII. p. 79.

23. Eukasiewicz. Lichen ruber acuminatus und planus an der Haut und Schleimhaut desselben Individuums und über die Identität des Lichen ruber acuminatus und der Pityriasis rubra pilaris. Arch. f. Derm. u. Syph. 1896. Bd. XXXIV. p. 163.

24. Crocker. a) Festschrift, gewidmet Pick. 1898. pag. 431. b) The British Journal of dermatology. 1890. Dec. 
25. Havas. a) Pityriasis rubra pilaris. Verhandlungen des Vereines Ungarischer Dermatologen und Urologen. Archiv f. Dermat. u. Syphilis. 1896. Bd. XXXVI. p. 245. - b) Ibidem. 1898. Bd. XLVI. p. 130.

26. L ew in. a) Pityriasis rubra pilaris. Verhandl, d. Berliner derm. Ges. Arch. f. Derm. u. Syph. 1895. Bd. XXXI. p. 267. - b) Ibidem. 1895. Bd. XXXII. p. 476. - c) Über Lichen ruber acuminatus und Pityriasis rubra pilaris. Verhandl. d. Berliner dermatol. Vereinigung. Ibidem. 1895. p. 205. - d) Ibidem. 1896. Bd. XXXV. p. 295.

27. Saalfeld. Ein Fall von atypischer Pityriasis rubra pilaris. Verh. d. Berl. derm. Vereinig. Arch. f. Derm. u. Syph. 1896. Bd. XXXVI. pag. 242 und 438.

28. Schloemann. Beitrag zur Lehre von der Pityriasis rubra pilaris Devergic. Inaug.-Diss. StralBburg. 1895. Ref. Arch. f. Dermatol. u. Syph. 1897. Bd. XXXIX. p. 278.

29. Török, a) Pityriasis rubra pilaris. Verhandlungen des Vereines ungarischer Dermatologen und Urologen. Archiv f. Derm. u. Syph. 1897. Bd. XXXVIII, pag. 464. - b) Mikroskopische Präparate über Pityriasis rubra pilaris. Arch. f. Derm. u. Syph. 1897. Bd. XL. p. 103. - c) Ibidem. 1898. Bd. XLII. p. 146.

30. Bredas. Beobachtungen und Betrachtungen über Lichen ruber. Festschrift, gewidmet Pick. 1898. p. 113.

31. Róna. Charakteristische Zeichen der Keratosis pilaris rubra und Pityriasis rubra pilaris (Devergie) an einem Individuum. Pester med. chir. Presse. 1898. Mai.

32. De Amicis. Un caso tipico di Pityriasis rubra pilaris. Società Italiana di Dermatologia e Sifilografia. Ref. Arch. f. Dermatol. u. Syph. 1906. Bd. LXXIX. p. 275.

33. Morton, Alexander. A case of pityriasis rubra pilaris (Devergie). Ref. Arch. f. Derm. u. Syph. 1899. Bd. L. p. 404.

34. Jordan. Ein Fall von Pityriasis rubra pilaris. Monatshefte f. prakt. Dermat. Bd. XXIV.

35. Fox. Ein Fall von Pityriasis rubra pilaris. Verhdl. d. Berliner dermat. Ges. Arch. f. Derm. u. Syph. 1899. Bd. L. p. 112.

36. Rosenthal. Ein Fall von Pityriasis rubra pilaris. Verhandl. der Berliner derm. Ges. Arch. f. Derm. u. Syph. 1899. Bd. XLVII. p. 295.

37. Pollitzer. Hin Fall von Pityriasis rubra pilaris. Verhandl. d. American Jermatological Association. Archiv f. Dermatol. u. Syph. 1899. Bd. XLIX. p. 396.

38. R a vogli. Pityriasis rubra pil. The Cincinati Lancet. 1899. Avril.

39. Dubreuilh. La pratique dermatologique. Bd. II.

40. Kalt. Pityriasis rubra pilaire. Soc. de Dermatol. et de Syphil. 1900. Paris. Decembre.

41. Meschtscherski. Moskauer venerologische und dermatolog. Gesellschaft. 1900. Dezember.

42. Palm. a) Ein Fall von Pityriasis rubra pilaris. Verhandlungen d. Berliner derm. Ges. Arch. f. Derm. u. Syph. 1900. Bd. LI. p. 433. - 
b) Demonstration desselben Kranken. 5. Januar 1904. Ibidem 1904. Band LXIX. pag. 436 .

43. Leredde. Pityriasis rubra pilaire. Soc. de Derm. et de Syph. Annal. de Dermatol. et de Syphil. 1898.

44. Pinkus. Mikroskopische Präparate über Pityriasis rubra pil. und Lichen ruber acuminatus. Verhandl. d. Berliner derm. Ges. Arch. f. Dermat. a. Syph. 1901. Bd. LVII. p. 274.

45. Du Castel et Kalt. Pityriasis rubra pilaire et lésions oculaires. Soc. de Dermat. et de Syph. Arch. f. Dermatol. u. Syphilis. 1902. Bd. LXIII. p. 446.

46. Hügel. Ein Fall von Pityriasis rubra pilaris (mikroskopische Präparate). Münchener mediz. Wochenschrift. 1900. Nr. 50.

47. Zieler. Ein Fall von Pityriasis rubra pilaris. Verhandlungen der Breslauer dermatol. Vereinigung. Archiv f. Dermatol. u. Syph. 1903. Bd. LXIV. p. 439.

48. A lexander. Pityriasis rubra pilaris. Verhandl. der Breslauer dermat. Vereinigung. Arch. f. Derm. u. Syph. 1903. Bd. LXIV. p. 419.

49. Hall, Arthur. Case of Pityriasis rubra pilaris in child of fours years. British Journal of Dermatology. 1903. Nr. 11.

50. Blaschko. Pityriasis rubra pilaris. Verhandl. der Berl. derm. Ges. Arch. f. Derm. u. Syph. 1904. Bd. LXIX. p. 227.

51. Sok olow. Zur Behandlung der Pityriasis rubra pilaris. Journ. russe de maladies cutan. et syphil. 1904. Juli. p. 39.

62. Thibierge. La pratique dermatologique. Bd. III.

53. Hudelo et Herisson. Pityriasis rubra pilaire chez un enfant de deux ans. Annal. de dermat. et syphil. 1905. p. 355.

54. Vignolo-Lutati. Über Pityriasis rubra pilaris. Arch. f. Derm. u. Syph. 1906. Bd. LXXIX. p. 273.

55. Cohn. Ein Fall von Pityriasis rubra pilaris. IX. Kongreß der Deutschen dermat. Gesellschaft in Bern. Arch. f. Dermat. u. Syph. 1906. Bd. LXXXII. p. 305.

56. Breda. Della Pityriasis rubra pilaris, Giorn. ital, delle malattie veneree e della pelle. 1907. Fasc. 5 . 